\title{
ASYMPTOTIC BEHAVIOUR AND PROPAGATION PROPERTIES OF THE ONE-DIMENSIONAL FLOW OF GAS IN A POROUS MEDIUM
}

BY

\author{
JUAN LUIS VAZQUEZ
}

\begin{abstract}
The one-dimensional porous media equation $u_{t}=\left(u^{m}\right)_{x x}, m>1$, is considered for $x \in R, t>0$ with initial conditions $u(x, 0)=u_{0}(x)$ integrable, nonnegative and with compact support. We study the behaviour of the solutions as $t \rightarrow \infty$ proving that the expressions for the density, pressure, local velocity and interfaces converge to those of a model solution. In particular the first term in the asymptotic development of the free-boundary is obtained.
\end{abstract}

0. Introduction. Suppose we have a certain distribution of gas whose density at time $t=0$ is given by a function $u_{0}(x)$ of one spatial direction $(x \in \mathbf{R})$. If the gas flows through a homogeneous porous medium the density $u=u(x, t)$ at time $t>0$ is governed by the equation

$$
u_{t}=\left(u^{m}\right)_{x x}
$$

for $x \in \mathbf{R}$ and $t>0$; $m$ is a physical constant, $m>1$, and we have scaled out other physical constants (see [1] for a physical derivation). $u$ satisfies the initial condition

$$
u(x, 0)=u_{0}(x)
$$

where $u_{0}$ satisfies the following assumptions:

$$
u_{0} \in L^{1}(\mathbf{R}), \quad u_{0} \geqslant 0, u_{0} \neq 0,
$$

and $u_{0}$ is compactly supported, i.e. if $\Omega_{0}=\left\{x \in \mathbf{R}: u_{0}(x)>0\right\}$ we have

$$
a_{1}=\text { ess inf } \Omega_{0}>-\infty, \quad a_{2}=\operatorname{ess} \sup \Omega_{0}<\infty \text {. }
$$

Sticking to the above application we define the pressure by $\nu=m u^{m-1} /(m-1)$ on $Q=\mathbf{R} \times(0, \infty)$ and the local velocity by $V=-\nu_{x}$ on the domain of dependence

$$
\Omega=\Omega[u]=\{(x, t) \in Q: u(x, t)>0\} .
$$

The total mass at time $t>0$ is $M(t)=\int u(x, t) d x$ and the center of mass is $x_{c}(t)=M(t)^{-1} \int u(x, t) x d x$. Set $M_{0}=\int u_{0}(x) d x$ and $x_{0}=M_{0}^{-1} \int u_{0}(x) x d x: M_{0}>0$ and $a_{1}<x_{0}<a_{2} . l_{0}=a_{2}-a_{1}$ measures the dispersion of the initial data.

Much is already known for problem (0.1)-(0.4); see [19] for a survey of results up to 1980 , where the $n$-dimensional case is considered, $n \geqslant 1$. In particular $(0.1)-(0.4)$

Received by the editors July 29, 1981 and, in revised form, February 1, 1982. This paper has been presented at the Symposium on Free-Boundary Problems Theory and Applications held in June, 1981, in Montecatini (Italy).

1980 Mathematics Subject Classification. Primary 35K65, 76S05, 35B40.

Key words and phrases. Flows in porous media, asymptotic behaviour, free boundaries, shiftingcomparison. 
admits a unique continuous weak solution $u(x, t) \geqslant 0$ [18], [3], such that for $t>0$, $u(\cdot, t)$ has compact support [14]. Thus $\Omega(t)=\{x \in \mathbf{R}: u(x, t)>0\}$ is bounded for every $t>0$ and two outer interfaces arise with equations $x=\zeta_{i}(t), i=1,2, t \geqslant 0$, where

$$
\begin{array}{lll}
\zeta_{1}(t)=\inf \Omega(t) & \text { if } t>0, & \zeta_{1}(0)=a_{1}, \\
\zeta_{2}(t)=\sup \Omega(t) & \text { if } t>0, & \zeta_{2}(0)=a_{2} .
\end{array}
$$

As a consequence of the inequality [10]

$$
u_{t} \geqslant-u /(m+1) t
$$

the set $\{\Omega(t): t>0\}$ is ordered by inclusion: $\Omega\left(t^{\prime}\right) \supset \Omega(t)$ if $t^{\prime}>t$ so that $(-1)^{i} \zeta_{i}$ is a nondecreasing function. Moreover there exist $t_{i}^{*} \geqslant 0$ (called waiting-times) such that for $0 \leqslant t \leqslant t_{i}^{*}, \zeta_{i}(t)=a_{i}[16]$ and, for $t>t_{i}^{*}, \zeta_{i}(t)$ is continuously differentiable and $(-1)^{i} \zeta_{i}^{\prime}(t)>0$ (once the interface starts to move it never stops) [11]. [16] proves that when $t \rightarrow \infty(-1)^{i} \zeta_{i}(t)$ behaves like $t^{1 /(m+1)}$. These results are proved under the simplifying hypotheses that $u_{0}$ is continuous, $u_{0}(x)>0$ for every $x \in I=\left(a_{1}, a_{2}\right)$ and vanishes outside $I$, but the proofs apply under conditions $(0.3),(0.4)$. On the contrary under only these two conditions the property that $\Omega(t)=\left(\zeta_{1}(t), \zeta_{2}(t)\right)$ does not hold in general, i.e. inner interfaces can appear that make $\Omega(t)$ disconnected for some time interval $0 \leqslant t \leqslant T, T>0$.

We shall be concerned in this paper with the following question: Give significant information about the behaviour of the solutions to (0.1)-(0.4) in terms of a simple information on $u_{0}$, specifically in terms of $M_{0}, x_{0}, a_{1}, a_{2}$. Our contribution deals with the asymptotic behaviour of density, pressure, velocity and free-boundaries and on the global properties of $\Omega$.

To describe the large-time behaviour we take as model solutions the class of explicit self-similar solutions corresponding to an initial "instantaneous source" given by Barenblatt in 1952 [6], i.e. solutions of $(0.1)$ with initial data

$$
u_{0}(x)=M \delta(x-a)
$$

where $M>0, a \in \mathbf{R}$ and $\delta$ is Dirac's delta function. The unique weak solution $\bar{u}(x, t ; M, a)$ is given in terms of its pressure by

$$
\vec{\nu}(x, t ; M, a)=\left[r(t)^{2}-(x-a)^{2}\right]_{+} / 2(m+1) t
$$

where

$$
\begin{aligned}
& r(t)=c_{m}\left(M^{m-1} t\right)^{1 /(m+1)} \\
& \text { with } c_{m}=\left\{\frac{2 m(m+1)}{m-1} B\left(\frac{m}{m-1}, \frac{1}{2}\right)^{1-m}\right\}^{1 /(m+1)},
\end{aligned}
$$

$B(\cdot, \cdot)$ being Euler's Beta function $\left([s]_{+}\right.$means $\left.\max (s, 0)\right)$. Its interfaces are the strictly monotone $C^{\infty}$-curves given by $\bar{\zeta}_{i}(t)=(-1)^{i} r(t)+a, i=1,2$, and the velocity is defined on $\Omega[\bar{u}]=\{(x, t) \in Q:|x-a|<r(t)\}$ by $\bar{V}(x, t)=(x-a)$. $((m+1) t)^{-1}$.

Let $\bar{u}(x, t ; M)=\bar{u}(x, t ; M, 0) ;$ then $\bar{u}(x, t ; M, a)=\bar{u}(x-a, t ; M)$. 
Our main result shows to what extent the solution $u(x, t)$ to $(0.1)-(0.4)$ resembles the self-similar $\bar{u}=\bar{u}\left(x, t ; M_{0}, x_{0}\right)$ with same mass and center of mass.

Theorem A. Let $u(x, t)$ be the solution to $(0.1)-(0.4)$ and let $\bar{u}=\bar{u}\left(x, t ; M_{0}, x_{0}\right)$. Then:

(i) for every $t \geqslant T^{*}=\left(l_{0} / c_{m}\right)^{m+1} M_{0}^{1-m}, \Omega(t)$ is the open interval $\left(\zeta_{1}(t), \zeta_{2}(t)\right)$ with strictly expanding borders;

(ii) as $t \rightarrow \infty$ we have

$$
\begin{gathered}
(-1)^{i}\left(\zeta_{i}(t)-\bar{\zeta}_{i}(t)\right) \downarrow 0 ; \\
\zeta_{i}^{\prime}(t) / \overline{\zeta_{i}^{\prime}}(t) \uparrow 1 \text { and } t\left|\zeta_{i}^{\prime}(t)-\overline{\zeta_{i}^{\prime}}(t)\right| \rightarrow 0 ; \\
t\left|V(x, t)-\left(x-x_{0}\right) /(m+1) t\right| \rightarrow 0 \quad \text { uniformly in } x \in \Omega(t), \\
t^{m /(m+1)}|\nu(x, t)-\bar{\nu}(x, t)| \rightarrow 0 \quad \text { uniformly in } x \in \mathbf{R}
\end{gathered}
$$

and for every $x \in \mathbf{R}, t>0$

$$
\nu(x, t) \leqslant \max _{x \in \mathbf{R}} \bar{\nu}(x, t)=c_{m}^{2}(2(m+1))^{-1}\left(M_{0}^{2} t^{-1}\right)^{(m-1) /(m+1)} .
$$

We may write $(0.10),(0.11)$ as giving the first term in the asymptotic development of $\zeta_{i}(t)$ and $\zeta_{i}^{\prime}(t)$ :

$$
\begin{gathered}
\zeta_{i}(t)=x_{0}+(-1)^{i} c_{m} M_{0}^{(m-1) /(m+1)} t^{1 /(m+1)}+o(1), \\
\zeta_{i}^{\prime}(t)=(-1)^{i}\left(c_{m} /(m+1)\right) M_{0}^{(m-1) /(m+1)} t^{m /(m+1)}+o(1 / t)
\end{gathered}
$$

where $o(1)$ and $o(1 / t)$ are the usual Landau $o$ 's taken as $t \rightarrow \infty$. Theorem A shows that $M_{0}$ and $x_{0}$ are the only relevant initial data in the first approximation to the large-time behaviour of the solutions to $(0.1)-(0.4)$. In particular $(0.13)$ implies for $1<m \leqslant 2$ the estimate

$$
t^{2 /(m+1)}|u(x, t)-\bar{u}(x, t)| \rightarrow 0
$$

uniformly in $x \in \mathbf{R}$. If $m>2$, however, (0.16) holds uniformly in $x:\left|x-x_{0}\right| \leqslant$ $\alpha r\left(t ; M_{0}\right)$ for every $0<\alpha<1$ and we obtain $t^{\sigma}|u(x, t)-\bar{u}(x, t)| \rightarrow 0$ at $t \rightarrow \infty$ uniformly in $x \in \mathbf{R}$ for $\sigma=m /\left(m^{2}-1\right)$.

As a precedent to these results Kamin [15] proved the convergence of $u$ towards a self-similar $\bar{u}$ with equal mass with an estimate

$$
t^{1 /(m+1)}|u(x, t)-\bar{u}(x, t)| \rightarrow 0
$$

that does not allow for the characterization of $x_{0}$. Friedman and Kamin [13] extend $(0.17)$ to dimensions $n \geqslant 1$. Several terms of the asymptotic representation of $u$ were stated in [7] without proof.

We begin by reviewing in $\$ 1$ several properties of the solutions. In particular we prove the time-invariance of the mass and the center of mass, i.e. for every $t>0$, $M(t)=M_{0}, x_{c}(t)=x_{0}($ Lemma 1.1).

In $\$ 2$ we introduce a comparison principle, based on the evaluation of masses, that we name "Shifting-Comparison Principle" (Sh.C.P.) (Lemma 3.2). As immediate corollaries we derive the estimate for the free boundaries $(-1)^{i} \zeta_{i}(t) t^{-1 /(m+1)}$ $\rightarrow c_{m} M_{0}^{(m-1) /(m+1)}$, which improves Knerr's result [16], and the estimate in Theorem 
A(i), where we remark that $T^{*}$ is optimal in terms of $M_{0}$ and $l_{0}$ as an upper bound for both the occurrence of waiting-times and that of an inner free-boundary.

$\S 3$ is devoted to proving Theorem A. As a main ingredient we use a sharp version of Caffarelli and Friedman's [11] differential inequality for the interfaces that in fact gives the monotonicity of $\zeta_{i}^{\prime}(t) t^{m /(m+1)}$ (Lemma 3.1).

The case where $u_{0}$ is a symmetric function is considered in $\S 4$. Then $u(x, t)$ is symmetric with respect to $x$ and we prove optimal rates of convergence in the results of Theorem A by means of a new "Concentration-Comparison Principle" (Theorem B).

Finally $\S 5$ considers the right interface $\zeta(t)$ of a solution of $(0.1),(0.2)$ with $u_{0}$ satisfying (0.3) and, instead of (0.4),

$$
\text { ess } \sup \Omega_{0}=0 \text {. }
$$

The behaviour of $\zeta$ as $t \rightarrow \infty$ and $t \rightarrow 0$ is investigated as well as its dependence on the $L^{p}$-norm of the initial data $u_{0}, 1 \leqslant p \leqslant \infty$.

Let us remark that the asymptotic behaviour of the porous medium equation in bounded domains of $\mathbf{R}^{n}$ has been studied recently by Aronson and Peletier [5].

The author is grateful to S. Kamin for comments and information on previous work and to the referee for several interesting remarks.

\section{Preliminaries.}

1.1 Existence of solutions. We begin by reviewing the existence and properties of weak solutions to $(0.1),(0.2)$. It is known [3] that for every $u_{0} \in L^{1}(\mathbf{R}), u_{0} \geqslant 0$, there exists a unique continuous function $u=u(x, t)$ in $Q=\mathbf{R} \times(0, \infty)$ with the following properties:

(i) $u \in C\left([0, \infty) ; L^{1}(\mathbf{R})\right) \cap L^{\infty}(\mathbf{R} \times[\delta, \infty))$ for every $\delta>0$,

(ii) $u_{t}=\left(u^{m}\right)_{x x}$ in the sense of distributions on $Q$,

(iii) $u(0)=u_{0}$ in $L^{1}(\mathbf{R})$.

Here $u(t)$ denotes the element $u(\cdot, t)$ in $L^{1}(\mathbf{R})$. Moreover $u_{t}$ and $\left(u^{m}\right)_{x x}$ exist a.e., $u_{t} \in L_{\mathrm{loc}}^{1}(Q)$ satisfies $(0.7)$ and

$$
\left\|u_{t}(\cdot, t)\right\|_{1} \leqslant(2 /(m+1) t)\left\|u_{0}\right\|_{1} .
$$

To obtain the solution we may approximate $u_{0}$ by a decreasing sequence of strictly positive, smooth functions $u_{0}^{n}$, apply to $u_{0}^{n}$ the existence theorem of [18] and derive (1.1) in the limit.

In $\$ 2$ we shall need an alternative approach: we discretize (0.1) in time and have recourse to Crandall and Liggett's Generation Theorem (see [12]). In fact given a continuous increasing function $\phi: \mathbf{R} \rightarrow \mathbf{R}, \phi(0)=0$, the operator $A=A_{\phi}$ defined on

$$
D(A)=\left\{u \in L^{1}(\mathbf{R}): \phi(u) \in W^{1, \infty}(\mathbf{R}) \text { and } \phi(u)_{x x} \in L^{1}(\mathbf{R})\right\}
$$

by

$$
A u=-\phi(u)_{x x} \text { if } u \in D(A)
$$


is $m$-accretive in $L^{1}(\mathbf{R})$, i.e. the resolvent $(I+\lambda A)^{-1}$ is a contraction on $L^{1}(\mathbf{R})$ for any $\lambda>0$ [9], and the closure of $D(A)$ in $L^{1}(\mathbf{R})$ is $L^{1}(\mathbf{R})$ [10]. Hence the formula

$$
S(t) u_{0}=\lim _{n \rightarrow \infty}(I+(t / n) A)^{-n} u_{0} \text { for } t \geqslant 0, u_{0} \in L^{1}(\mathbf{R}),
$$

defines a semigroup of contractions $S(t)=S_{\phi}(t), t \geqslant 0$, in $L^{1}(\mathbf{R})$. Bénilan has proved [8] that $u(x, t)=\left(S(t) u_{0}\right)(x)$ solves in a generalized sense (called integral or mild sense) the evolution problem

$$
\left\{\begin{array}{l}
u_{t}=\phi(u)_{x x}, \quad x \in \mathbf{R}, t>0 \\
u(x, 0)=u_{0}(x) \in L^{1}(\mathbf{R})
\end{array}\right.
$$

and that these mild solutions are unique.

Setting $\phi(t)=s|s|^{m-1}$ we recover problem $(0.1),(0.2)$ and both constructions give the same unique solution satisfying (1.1).

Since for every $f_{i} \in L^{\mathrm{l}}(\mathbf{R}), i=1,2$, and $\lambda>0$ we have [9]

$$
\left\|\left[(I+\lambda A)^{-1} f_{1}-(I+\lambda A)^{-1} f_{2}\right]_{+}\right\|_{1} \leqslant\left\|\left[f_{1}-f_{2}\right]_{+}\right\|_{1},
$$

using (1.5) we obtain for every $u_{i} \in L^{\mathrm{l}}(\mathbf{R}), i=1,2$,

$$
\left\|\left[S(t) u_{1}-S(t) u_{2}\right]_{+}\right\|_{1} \leqslant\left\|\left[u_{1}-u_{2}\right]_{+}\right\|_{1} .
$$

(1.7) implies obvious comparison results for the solutions of $(0.1),(0.2)$.

REMARK. Since the solutions $\bar{u}(x, t ; M, a)$ are limits of solutions of $(0.1)$ with initial data $u_{0}^{n} \geqslant 0, u_{0}^{n} \in L^{1}(\mathbf{R})$, such that $u_{0}^{n} \rightarrow M \delta(x)$ in the $w^{*}$-topology of $\mathscr{N}(\mathbf{R})$, the space of bounded Radon measures (take $u_{0}^{n}(x)=\bar{u}(x, 1 / n ; M, a)$ ), the comparison results valid for solutions with $L^{1}$-data apply also to $\bar{u}$.

1.2. Group of transformations. Equation (0.1) admits the biparametric group of transformations

$$
\hat{u}=k u, \quad \hat{x}=L^{-1} x, \quad \hat{t}=k^{1-m} L^{-2} t,
$$

i.e. if $u(x, t)$ is a solution of $(0.1)$ with initial condition $u_{0}(x)$ then for every $k, L>0, \hat{u}(x, t)$ defined by

$$
\hat{u}(x, t)=k u\left(L x, L^{2} k^{m-1} t\right)
$$

is a solution with initial condition $\hat{u}_{0}(x)=k u_{0}(L x)$. We write $\hat{u}=T_{K, L} u$. The transformation $T_{K, L}$ preserves the (initial) mass iff $k=L$.

We can use the group of transformations $T_{K, L}$ to reduce a problem $(0.1),(1.2)$ to a simple one. Thus if $u(x, t)$ is such that $\int u_{0}(x) d x=M$ and we put $u=T_{M, 1} \hat{u}$ we have $\hat{M}=\int \hat{u}_{0}(x) d x=1$. By means of $T_{M, 1}$ our conclusions on $\hat{u}$ apply to $u$. For instance the respective free boundaries $\zeta(t)$ and $\hat{\zeta}(t)$ are related by

$$
\zeta(t)=\hat{\zeta}\left(M^{m-1} t\right)
$$

Notice that the self-similar solutions $\bar{u}(x, t ; M)$ centered at $a=0$ are invariant under $T_{k, k}, k>0$, i.e.

$$
\bar{u}(x, t ; M)=k \bar{u}\left(k x, k^{m+1} t ; M\right) .
$$


1.3. Two invariants. We establish here the invariance of the total mass $M$ and the center of mass $x_{c}$ for the solutions of (0.1)-(0.4):

LEMMA 1.1. For every $t>0 M(t)=M_{0}$ and $x_{c}(t)=x_{0}$.

Proof. Assume first that $u_{0}$ is also continuous, positive on $I=\left(a_{1}, a_{2}\right)$ and such that $u_{0}(x)>\left|x-a_{i}\right|^{1 /(m-1)}$ in a neighbourhood of both $a_{1}$ and $a_{2}$ (so that the waiting-times $t_{1}^{*}$ and $t_{2}^{*}$ vanish, see [16]). Then $u \in C^{\infty}(\Omega)$ and $\nu \in C^{1}(\bar{\Omega} \cap Q)$, see [11]. It follows that $u^{m} \in C^{1}(\bar{\Omega} \cap Q)$ and $\left(u^{m}\right)_{x}$ vanishes on both interfaces.

Take now two arbitrary times $t_{2}>t_{1}>0$ and set $G=\left\{(x, t): t_{1} \leqslant t \leqslant t_{2}\right.$ and $\left.\zeta_{1}(t) \leqslant x \leqslant \zeta_{2}(t)\right\}$. Then

$$
\begin{aligned}
\int_{\mathbf{R}} u\left(x, t_{2}\right) x d x-\int_{\mathbf{R}} u\left(x, t_{1}\right) d x & =\iint_{G} u_{t} d x d t \\
& =\int_{t_{1}}^{t_{2}} d t\left[\left(u^{m}\right)_{x}\left(\zeta_{2}(t), t\right)-\left(u^{m}\right)_{x}\left(\zeta_{1}(t), t\right)\right]=0, \\
\int_{\mathbf{R}} u\left(x, t_{2}\right) x d x-\int_{\mathbf{R}} u\left(x, t_{1}\right) x d x & =\iint_{G} x u_{t} d x=\int_{t_{1}}^{t_{2}} d t\left[x\left(u^{m}\right)_{x}-u^{m}\right]_{\left(\zeta_{1}(t), t\right)}^{\left(\zeta_{2}(t), t\right)}=0,
\end{aligned}
$$

and the result follows. For general $u_{0}$ approximate by a decreasing sequence $\left\{u^{n}\right\}$ as above and pass to the limit using the $L^{1}$-continuity of the map $u_{0} \mapsto u(t)$ (formula (1.7)). \#

REMARKS. (1) The result is valid in a much more general context: for instance for the solutions of $\left(P_{\phi}\right)$, without the restriction of nonnegativity.

(2) The invariance of the total mass has been widely used in connection with this problem: $[10,15], \ldots$ The invariance of the center of mass has been pointed out in [7].

1.4. Regularity up to the interfaces. We know that the solutions are classical in $\Omega$. [1] proves that $\nu(x, t)$ is Lipschitz-continuous in $x$ in $Q_{\tau}=\mathbf{R} \times(\tau, \infty)$ for every $\tau>0$. But $\nu_{x}$ need not be continuous at the interfaces (check the self-similar solutions). However [16] proves that $\nu_{x}\left(\zeta_{i}(t), t\right)$ exists for every $t>0$ as the limit of $\nu_{x}(x, t), x \rightarrow \zeta_{i}(t), x \in \Omega(t)$ and

$$
V\left(\zeta_{i}(t), t\right) \equiv-\nu_{x}\left(\zeta_{i}(t), t\right)=\zeta_{i}^{\prime}(t)
$$

where $\zeta_{i}^{\prime}(t)$ is the right derivative of $\zeta$ at $t$. [11] proves that $\nu_{x}$ is continuously differentiable up to the boundary $x=\zeta_{i}(t)$ if $t>t_{i}^{*}$.

\section{Comparison by shifting.}

2.1. We introduce in this section a "Shifting-Comparison Principle" that allows us to compare a solution with given initial condition with the one corresponding to a displaced initial condition. To measure the relative displacement we use the corresponding distribution functions defined by

$$
U(x, t)=\int_{-\infty}^{x} u(x, t) d x=\text { amount of mass in }(-\infty, x] .
$$

The idea behind the principle is that it is more feasible to compare masses than to compare point densities. The principle is in fact a maximum principle for the "integrated" equation $U_{t}=\left(U_{x}\left|U_{x}\right|^{m-1}\right)_{x}$. 
We prove the principle in an elliptic version. Then (1.5) allows us to derive the evolution version.

Lemma 2.1 (Shifting Comparison Principle. Elliptic Version). Let $\beta$ be $a$ continuous nondecreasing function such that $0=\beta(0) \subset \operatorname{Int} \beta(\mathbf{R})$ and let $f_{i}, i=1,2$, be integrable functions such that for every $x \in \mathbf{R}$,

$$
\int_{-\infty}^{x} f_{1}(x) d x \leqslant \int_{-\infty}^{x} f_{x}(x) d x .
$$

Let $u_{i}$ be the solution $(E):-u^{\prime \prime}+\beta(u)=f_{i}$, with $u_{i} \in W^{1, \infty}(\mathbf{R})$ and $w_{i}=\beta\left(u_{i}\right) \in$ $L^{1}(\mathbf{R})$ (see [9]). Then for every $x \in \mathbf{R}$,

$$
\int_{-\infty}^{x} w_{1}(x) d x \leqslant \int_{-\infty}^{x} w_{2}(x) d x .
$$

Proof. Set $F_{i}(x)=\int_{-\infty}^{x} f_{i}(x) d x$ and $W_{i}(x)=\int_{-\infty}^{x} w_{i}(x) d x$. Assume that $(2.3)$ does not hold so that $G=\left\{x \in \mathbf{R}: W_{1}(x)>W_{2}(x)\right\}$ is nonvoid. Let $I=(a, b)$, $-\infty \leqslant a<b \leqslant \infty$, be a maximal interval in $G$. For every $x \in I$ we have by integration of $(E)$ :

$$
u_{1}^{\prime}(x)=W_{1}(x)-F_{1}(x)>W_{2}(x)-F_{2}(x)=u_{2}^{\prime}(x)
$$

so that $u_{1}-u_{2}$ is strictly increasing on $I$.

Assume now that $a>-\infty$. Then by continuity $W_{1}(a)=W_{2}(a)$ and $u_{1}(a)>u_{2}(a)$ (if $u_{1}(a)<u_{2}(a)$ we would have $u_{1}(x)<u_{2}(x)$ if $|x-a|<\varepsilon$ for an $\varepsilon>0$ so that $w_{1}(x) \leqslant w_{2}(x)$, hence $W_{1}-W_{2}$ is nonincreasing in $a-\varepsilon<x<a+\varepsilon$, contradicting the definition of $a$ ). By (2.4) we have $u_{1}>u_{2}$ on $I$, so that $W_{1}-W_{2}$ is nondecreasing on $I$. This implies that $b=\infty$ and $W_{1}(\infty)>W_{2}(\infty)$. But this contradicts the fact that $F_{1}(\infty) \leqslant F_{2}(\infty)$ and that $W_{i}(\infty)=F_{i}(\infty)$ for the solutions of $(E)$ (see [9, formula (4.3)]).

If $a=-\infty, W_{i}(-\infty)=0$ and $u_{1}(-\infty) \geqslant u_{2}(-\infty)$ by the preceding argument. Hence the same conclusion holds. \#

REMARK. It is clear that the proof of Lemma 2.1 applies to much more general situations. In particular it is true for the solutions of

$$
-\frac{d}{d x} A\left(x, u^{\prime}(x)\right)+B(x, u(x))=f(x)
$$

where $A$ and $B$ are, say, increasing in $u$ and continuous in $x$, and $f \in L^{1}(\mathbf{R})$.

Besides it holds for suitable Dirichlet or Neumann boundary conditions if (2.5) is posed in a bounded interval or a half-line.

Similar remarks apply to Lemma 2.2 to follow. \#

The change of variables $w=\beta(u), u=\phi(w)$ transforms $-u^{\prime \prime}+\beta(u)=f$ into $-\phi(w)^{\prime \prime}+w=f$, that can be written as $w=\left(I+A_{\phi}\right)^{-1}(f)$. Therefore setting $\phi(s)$ $=s|s|^{m-1}$, i.e. $\beta(s)=s|s|^{(1-m) / m}$, we derive via (1.5) the following evolution version for the solutions of $(0.1),(0.2)$ :

Lemma 2.2 (Shifting-Comparison Principle. Parabolic version). Let $u^{1}(x, t)$, $u^{2}(x, t)$ be solutions of $(0.1),(0.2)$ with initial data $u_{0}^{1}(x), u_{0}^{2}(x) \in L^{1}(\mathbf{R})$. If for every 
$x \in \mathbf{R}$ we have

$$
\int_{-\infty}^{x} u_{0}^{1}(x) d x \leqslant \int_{-\infty}^{x} u_{0}^{2}(x) d x, \text { i.e. } U_{0}^{1}(x) \leqslant U_{0}^{2}(x),
$$

then for every $t>0$ and every $x \in \mathbf{R}$,

$$
\int_{-\infty}^{x} u^{1}(x, t) d x \leqslant \int_{-\infty}^{x} u^{2}(x, t) d x, \text { i.e. } \quad U^{1}(x, t) \leqslant U^{2}(x, t) .
$$

REMARKS. (1) We say that the mass of $u^{1}$ is shifted to the right with respect to that of $u^{2}$ at time $t=0$; this situation is preserved for every $t>0$.

(2) As said in $\S 1.1$ we may consider initial data of the form $M \delta\left(x-x_{0}\right)$.

2.2. First applications. An easy application of the Sh.C.P., comparing with selfsimilar solutions that concentrate all the mass $M_{0}$ of $u_{0}$ at the extreme points $x=a_{1}$ or $x=a_{2}$, allows us to bound the right and left interface of $u$ from above and below, giving a first estimate of their asymptotic behaviour. Here and in the sequel we fix $M=M_{0}$ in $r(t): r(t)=c_{m}\left(M_{0}^{m-1} t\right)^{1 /(m+1)}$ :

COROllary 2.3. For every $t>0$ we have

$$
a_{1}-r(t)<\zeta_{1}(t)<a_{2}-r(t), \quad a_{1}+r(t)<\zeta_{2}(t)<a_{2}+r(t),
$$

so that

$$
\lim _{t \rightarrow \infty} \zeta_{i}(t) t^{-1 /(m+1)}=(-1)^{i} c_{m} M_{0}^{(m-1) /(m+1)} .
$$

Proof. We take $u^{1}(x, t)=\bar{u}\left(x, t ; M_{0}, a_{1}\right)$ and $u^{2}(x, t)=\bar{u}\left(x, t ; M_{0}, a_{2}\right)$. Since we have $U_{0}^{1}(x) \geqslant U_{0}(x) \geqslant U_{0}^{2}(x)$, we conclude that $U^{1}(x, t) \geqslant U(x, t) \geqslant U^{2}(x, t)$. But since the interfaces can be characterized in terms of $U: \zeta_{1}(t)=$ $\inf \{x \in \mathbf{R}: U(x, t)>0\}, \zeta_{2}(t)=\sup \left\{x \in \mathbf{R}: U(x, t)<M_{0}\right\}$, it follows that $\zeta_{1}^{1}(t)=$ $a_{1}-r(t) \leqslant \zeta_{1}(t) \leqslant \zeta_{1}^{2}(t)=a_{2}-r(t)$ and likewise for $\zeta_{2}(t)$.

We must prove that the inequalities (2.8) are strict: assume for instance that for a $t_{0}>0, \zeta_{2}\left(t_{0}\right)=a_{2}+r\left(t_{0}\right)$. Since $\zeta_{2}(t) \leqslant a_{2}+r(t)$ for every $t>0$, we have $\zeta_{2}^{\prime}\left(t_{0}\right)$ $=r^{\prime}\left(t_{0}\right)$. Take now $\tilde{u}(x, t)=\bar{u}\left(x-a_{2}, t ; M_{0}\right)$. For $t=t_{0}$ we have $\zeta_{2}\left(t_{0}\right)=\tilde{\zeta}_{2}\left(t_{0}\right)$, $\zeta_{2}^{\prime}(t)=\tilde{\zeta}_{2}^{\prime}(t)$. Using the fact that $\nu_{x}(\zeta(t), t)=-\zeta^{\prime}(t+)$ for any $t>0$ [16], and that $\nu_{x x} \geqslant 0-1 /(m+1) t=\tilde{\nu}_{x x}$ (in $\left.\Omega[\tilde{u}]\right)$ [3], we conclude that, at $t=t_{0}, u\left(x, t_{0}\right) \geqslant$ $\tilde{u}\left(x, t_{0}\right)$. Since both have mass $M_{0}$ it follows that $u=\tilde{u}$ at $t=t_{0}$. But this is impossible since $x_{0}<a_{2}=\tilde{x}_{0}$ and the center of mass is invariant. \#

As a consequence of (2.8) we bound above the waiting-times $t_{i}^{*}$ in terms of $M_{0}=\left\|u_{0}\right\|_{1}$ and $l_{0}=a_{2}-a_{1}$ :

\section{COROLlary 2.4. We have}

$$
t_{i}^{*}<T^{*} \equiv\left(l_{0 / c_{m}}\right)^{m+1} M_{0}^{1-m} .
$$

Proof. For $t \geqslant T^{*}, \zeta_{2}(t)>a_{1}+r\left(T^{*}\right)=a_{1}+l_{0}=a_{2}$. Similarly for $\zeta_{1}$. \#

REMARKS. (1) (2.10) is sharp in terms of $M_{0}$ and $l_{0}$. To see this choose an initial datum $u_{0}$ with two components: one, $u_{0}^{1}(x)$, of mass $\geqslant M_{0}-\varepsilon, \varepsilon>0$ small, supported in $\left[a_{1}, a_{1}+\varepsilon\right]$, and the other, $u_{0}^{2}(x)$, of mass obviously $\leqslant \varepsilon$, supported in $\left[a_{1}-\varepsilon, a_{2}\right]$, and such that the corresponding solution $u^{2}(x, t)$ has vertical interfaces for at least a time $T^{*}$. Up to the time where the interfaces of the solutions to both 
partial initial data, $u^{1}(x, t)$ and $u^{2}(x, t)$, meet we have $u(x, t)=u^{1}(x, t)+u^{2}(x, t)$ so that $\zeta(t)=a_{2}$. But as $\varepsilon \rightarrow 0$ this time is easily seen to approach $T^{*}$ (use the Sh.C.P.).

(2) For recent work on the determination of the waiting-times see [4 and 17]. \#

We now turn to the existence of an inner free-boundary, $\Gamma_{\mathrm{in}}$. Since we are mainly interested in the large-time behaviour we want to bound above the time at which the inner free-boundary ceases to exist. For that we define on $\mathbf{R}$ the nonnegative function

$$
\tau(x)=\sup \{t>0: u(x, t)=0\} .
$$

It is clear that $\Omega[u]=\{(x, t) \in \mathbf{Q}: t>\tau(x)\}$. We have as a conseqence of Corollary 2.4 :

COROLlaRY 2.5. For every $x \in\left[a_{1}, a_{2}\right], \tau(x)<T^{*}$ so that

$$
\Gamma_{\text {in }} \subset\left(a_{1}, a_{2}\right) \times\left(0, T^{*}\right) .
$$

Proof. If $x=a_{1}$ or $x=a_{2}, \tau\left(a_{i}\right)=t_{i}^{*}$ and we are reduced to (2.10). For any $\bar{x}$ : $a_{1}<\bar{x}<a_{2}$ such that $\tau(\bar{x})>0$, we write $u_{0}(x)$ as $u_{0}=u_{0}^{(1)}+u_{0}^{(2)}$ with $u_{0}^{(1)}=$ $u_{0} \cdot \chi_{(-\infty, \bar{x}]}$ and $u_{0}^{(2)}=u_{0} \cdot \chi_{(\bar{x}, \infty)}, \chi_{E}$ denoting the characteristic function of a set $E \subset \mathbf{R}$. Let $u^{(1)}, u^{(2)}$ be the respective solutions. By comparison $u(x, t) \geqslant u^{(i)}(x, t)$ so that $\tau(\bar{x}) \leqslant \tau^{(i)}(\bar{x}), i=1,2$.

Now observe that $\tau^{(1)}(\bar{x})=t_{2}^{*(1)}=$ the right waiting-time of $u^{(1)}$ and $\tau^{(2)}(\bar{x})=$ $t_{1}^{*(2)}=$ the left waiting-time of $u^{(2)}$, so that

$$
\tau(\bar{x})<\left(\frac{\bar{x}-a_{1}}{c_{m}}\right)^{m+1} M_{1}^{1-m} \quad \text { with } M_{1}=\int_{a_{1}}^{\bar{x}} u_{0}(x) d x
$$

and

$$
\tau(\bar{x})<\left(\frac{a_{2}-\bar{x}}{c_{m}}\right)^{m+1} M_{2}^{1-m} \text { with } M_{2}=M_{0}-M_{1} .
$$

It follows from (2.13), (2.14) that

$$
\begin{aligned}
l_{0} & =\left(a_{2}-\bar{x}\right)+\left(\bar{x}-a_{1}\right)>c_{m}\left(M_{1}^{(m-1) /(m+1)}+M_{2}^{(m-1) /(m+1)}\right) \tau(\bar{x}) \\
& \geqslant c_{m} M_{0}^{(m-1) /(m+1)} \tau(\bar{x})
\end{aligned}
$$

and the result follows. \#

REMARKS. (1) (2.12) is sharp: argue as in Remark (1) to Corollary 2.2.

(2) It is not difficult to see that

$$
\Gamma_{\text {in }}=\{(x, \tau(x)): x \in \operatorname{Int}(\Lambda)\} \cup\{(x, t): x \in \partial \Lambda \cap \Lambda \text { and } 0<t \leqslant \tau(x)\}
$$

where $\Lambda=\left\{\left(a_{1}, a_{2}\right): \tau(x)>0\right\}$ and that for every maximal open interval $I$ in $\Lambda$, $\Gamma_{\text {in }} \cap(I \times(0, \infty))$ consists of one or two monotone $C^{1}$-arcs (pieces of interfaces to subsolutions as above). \#

3. The asymptotic behaviour. This section is devoted to proving Theorem A (ii): the fact that $\max _{x \in \mathbf{R}} u(x, t) \leqslant \max _{x \in \mathbf{R}} \bar{u}\left(x, t ; M_{0}\right)$ for every $t>0$ follows easily 
from two properties: (i) $u$ and $\bar{u}$ have the same mass, $M_{0}$; (ii) $\nu_{x x} \geqslant-((m+1) t)^{-1}$ in $\Omega[u], \bar{v}_{x x}=-((m+1) t)^{-1}$ in $\Omega[\bar{u}]$.

The rest is based on a precise description of the outer interfaces. We begin by revisiting the second-order differential inequality for the $\zeta_{i}$ 's obtained by Caffarelli and Friedman [11]:

LEMMA 3.1. There exist nonnegative measures $\mu_{i}, i=1,2$, on $(0, \infty)$ such that

$$
\zeta_{i}^{\prime \prime}(t)+(m /(m+1) t) \zeta_{i}^{\prime}(t)=\mu_{i}(t)(-1)^{i}
$$

in the sense of distributions. Hence the expression $(-1)^{i} \zeta_{i}^{\prime}(t) t^{m /(m+1)}$ is nondecreasing in $(0, \infty){ }^{1}$

REMARKS. (1) The coefficient $m /(m+1)$ is best possible: the self-similar solutions $\bar{u}(x, t ; M, a)$ satisfy $(3.1)$ with $\mu_{i}=0$.

(2) Caffarelli and Friedman's result states (3.1) in the form $\zeta_{i}^{\prime \prime}(t)+k \zeta_{i}^{\prime}(t)=$ $\mu_{i}(t)(-1)^{i}$ with a constant $k>0$. But the specification of $k$ as $m /(m+1) t$ plays a fundamental role in the sequel for it gives the monotonicity of $\zeta^{\prime}(t) t^{m /(m+1)}$.

Proof. We review the proof in [11] to point out how $k$ may be replaced by $m /(m+1) t$.

Let us take the case $i=2$ and drop the $i$ for simplicity. At a point of the interface $\left(\zeta\left(t_{0}\right), t_{0}\right)$ with $t_{0}>t^{*}$, we adapt a self-similar solution $\tilde{u}=\bar{u}\left(x-x_{1}, t ; M_{1}\right)$ with $x_{1}, M_{1}$ so chosen as to have (i) $\zeta\left(t_{0}\right)=\tilde{\zeta}(t)$ and (ii) $\zeta^{\prime}\left(t_{0}\right)=\tilde{\zeta}^{\prime}\left(t_{0}\right)$, i.e. $\nu_{x}\left(\zeta\left(t_{0}\right), t_{0}\right)$ $=\tilde{\nu}_{x}\left(\zeta\left(t_{0}\right), t_{0}\right)$. Since $\nu_{x x} \geqslant-((m+1) t)^{-1}=\tilde{\nu}_{x x}$ in $\Omega[\tilde{u}]$, it follows for $t \geqslant t_{0}$ that $u(x, t) \geqslant \tilde{u}(x, t)$. As in [11] we conclude that for $h>0$,

$$
\zeta\left(t_{0}+h\right)-\zeta\left(t_{0}\right)-\zeta^{\prime}\left(t_{0}\right) h \geqslant \tilde{\zeta}\left(t_{0}+h\right)-\tilde{\zeta}\left(t_{0}\right)-\zeta^{\prime}\left(t_{0}\right) h .
$$

Using the fact that for the self-similar solution $\tilde{u}$,

$$
\tilde{\zeta}^{\prime \prime}\left(t_{0}\right)+\left(m /(m+1) t_{0}\right) \tilde{\zeta}^{\prime}\left(t_{0}\right)=0,
$$

the second-member of (3.2) equals $-\left(h^{2} / 2\right) \cdot\left(m /(m+1) t_{0}\right) \zeta^{\prime}\left(t_{0}\right)+O\left(h^{3}\right)$.

Now define the function $\Phi_{h}$ for $h>0$ fixed by

$$
\Phi_{h}\left(t_{0}\right)=\frac{\zeta\left(t_{0}+h\right)-\zeta\left(t_{0}\right)-h \zeta^{\prime}\left(t_{0}\right)}{h^{2} / 2} \geqslant-\frac{m}{(m+1) t_{0}} \zeta^{\prime}\left(t_{0}\right)+O(h) .
$$

One proves as in [11] that a subsequence of $\Phi_{h}$ converges weakly towards a signed measure so that in the limit (3.5) gives (dropping the zeros)

$$
\zeta^{\prime \prime}(t)+(m /(m+1) t) \zeta^{\prime}(t)=\mu
$$

in the distribution sense in $\left(t^{*}, \infty\right)$ and $\mu$ is nonnegative. Now divide (3.5) by $\zeta^{\prime}(t)>0$ to get

$$
\lg \left(\zeta^{\prime}(t) t^{m /(m+1)}\right)^{\prime}=\mu(t) / \zeta^{\prime}(t) \geqslant 0 .
$$

Therefore $\zeta^{\prime}(t) t^{m /(m+1)}$ is nondecreasing in $\left(t^{*}, \infty\right)$. But since $\zeta(t)=a_{2}$ in $0 \leqslant t \leqslant t^{*}$, the assertions of the lemma hold in $(0, \infty)$. \#

\footnotetext{
${ }^{1}$ If $t=t_{i}^{*}, \zeta_{i}^{\prime}\left(t_{i}^{*}\right)$ means the right derivative $\zeta_{i}^{\prime}\left(t_{i}^{*}+\right)$.
} 
We recall that for the self-similar solution $\bar{u}=\bar{u}\left(x, t ; M_{0}\right)$, the interfaces are given by $(-1)^{i} \bar{\zeta}_{i}(t)=r(t) \equiv c_{m}\left(M_{0}^{m-1} t\right)^{1 /(m+1)}$. Combining Corollary 2.3 and Lemma 3.1 we obtain

LEMMA 3.2. For $t>t_{i}^{*}$ we have

$$
(-1)^{i} \zeta_{i}^{\prime}(t) \leqslant r^{\prime}(t) \text { and }(-1)^{i} \zeta_{i}^{\prime}(t) / r^{\prime}(t) \uparrow 1 \text { as } t \rightarrow \infty .
$$

There exist $b_{i}, i=1,2$, such that $a_{1} \leqslant b_{i} \leqslant a_{2}$, and as $t \rightarrow \infty$,

$$
\zeta_{2}(t)-r(t) \downarrow b_{2}, \quad \zeta_{1}(t)+r(t) \uparrow b_{1} .
$$

Finally

$$
\lim _{t \rightarrow \infty} t\left|\zeta_{i}^{\prime}(t)-(-1)^{i} r^{\prime}(t)\right|=0 .
$$

Proof. We may consider only the case $M_{0}=1$ and $i=2$. Also we drop the $i$ 's.

Since $\zeta^{\prime}(t) t^{m /(m+1)}$ is nondecreasing (Lemma 3.1) there exists the limit $\lim _{t \rightarrow \infty} \zeta^{\prime}(t) t^{m /(m+1)}=K \leqslant \infty$. Since $\lim _{t \rightarrow \infty} \zeta(t) t^{-1 /(m+1)}=c_{m}$ (Corollary 2.3) we conclude that $K=c_{m} /(m+1)$ and (3.7) is proved.

In particular we have $\zeta^{\prime}(t) \leqslant r^{\prime}(t)$ so that $\eta(t)=\zeta(t)-r(t)$ is nonincreasing in $t$. Since $a_{1}<\eta(t)<a_{2}$, (3.8) follows.

To prove (3.9) write (3.1) in the form

$$
\left(t \zeta^{\prime}\right)^{\prime}=\zeta^{\prime} /(m+1)+t \mu(t) .
$$

Integrating in $t$ gives

$$
t \zeta^{\prime}(t)=\frac{\zeta(t)-a}{m+1}+\int_{0}^{t} t d \mu(t)
$$

Let $\xi(t)=\int_{0}^{t} t d \mu(t)$. (3.11) can be written as $t \eta^{\prime}(t)=\left(\eta(t)-a_{2}\right) /(m+1)+\xi(t)$ so that (3.9) is equivalent to $\xi(\infty)=\left(a_{2}-b_{2}\right) /(m+1)$. If this is not true and, say, $\xi(\infty) \geqslant\left(a_{2}-b_{2}\right) /(m+1)+\varepsilon$ for an $\varepsilon>0$, then we would have $\lim t \eta^{\prime}(t) \geqslant \varepsilon$ as $t \rightarrow \infty$ so that $\lim \eta(t) / \lg t \geqslant \varepsilon$. But since $|\eta(t)| \leqslant \max \left(\left|a_{1}\right|,\left|a_{2}\right|\right)$, this is not possible. The same argument holds if $\xi(\infty)<\left(a_{2}-b_{2}\right) /(m+1)$. \#

REMARK. (3.11) implies that $(-1)^{i}\left(\zeta_{i}(t)-a_{i}\right) t^{-1 /(m+1)}$ is monotone nondecreasing. Hence we can formulate (2.9) more precisely:

$$
(-1)^{i}\left(\zeta_{i}(t)-a_{i}\right) t^{-1 /(m+1)} \uparrow c_{m} M_{0}^{(m-1) /(m+1)} . \quad \#
$$

To obtain the asymptotic expression $(0.10)$ for $\zeta_{i}(t)$ we need yet to show that $b_{1}=b_{2}=x_{0}$. We introduce the following expression: $d(t)=l(t)-2 r(t)$, where $l(t)=\zeta_{1}(t)+\zeta_{2}(t)$ is the dispersion of $u$ at time $t$. (3.7) says that $d^{\prime}(t) \leqslant 0$ and (3.8) that $d(t) \downarrow b_{2}-b_{1}$ as $t \rightarrow \infty$. We show next that $b_{1}=b_{2}$ :

LEMMA 3.3. $b_{1}=b_{2}$, i.e., there exists $b \in\left(a_{1}, a_{2}\right)$ such that as $t \rightarrow \infty$,

$$
\zeta_{i}(t)=(-1)^{i} r(t)+b+o(1) .
$$

Proof. We divide the proof in two parts.

(I) We prove first that $b_{2} \geqslant b_{1}$, i.e. that $\lim _{t \rightarrow \infty} d(t) \geqslant 0$. For that we evaluate $\nu$ at a fixed $t>0$. Since $\nu\left(\zeta_{1}(t), t\right)=0$,

$$
\nu_{x}\left(\zeta_{1}(t), t\right)=-\zeta_{1}^{\prime}(t)=(r(t)-\varepsilon(t)) /(m+1) t
$$


where $\varepsilon(t) \geqslant 0, \varepsilon(t) \rightarrow 0$ as $t \rightarrow \infty$, and $\nu_{x x} \geqslant((m+1) t)^{-1}$, we have

$$
\nu(x, t) \geqslant-\frac{\hat{x}^{2}}{2(m+1) t}+\frac{(r(t)-\varepsilon(t)) \hat{x}}{(m+1) t} \quad \text { if } \hat{x} \geqslant 0,
$$

where $\hat{x}=x-\zeta_{1}(t)$. Hence $\nu(x, t)=0, x>\zeta_{1}(t)$ implies $\hat{x} \geqslant 2(r(t)-\varepsilon(t))$. But since $\nu(x, t)=0$ for $x=\zeta_{2}(t)$, i.e. for $\hat{x}=l(t)=2 r(t)+d(t)$, we conclude that $d(t) \geqslant-2 \varepsilon(t)$. Let $t \rightarrow \infty$ to conclude.

(II) We now prove that $b_{1}>b_{2}$ cannot occur.

The idea is to compare $u$ at fixed times $t \gg 0$ with the self-similar solutions $\tilde{u}=\bar{u}\left(x-x^{*}(t), t ; M_{0}\right), x^{*}(t)=\frac{1}{2}\left(\zeta_{1}(t)+\zeta_{2}(t)\right)$ (i.e. the one centered in $\left.\Omega(t)\right)$ and estimate the integrals at time $t$ :

$$
\begin{aligned}
& I_{1}(t)=\int_{[u<\tilde{u}]}(\tilde{u}(x, t)-u(x, t)) d x, \\
& I_{2}(t)=\int_{[u>\tilde{u}]}(u(x, t)-\tilde{u}(x, t)) d x .
\end{aligned}
$$

Since $\int u(x, t) d x=\int \tilde{u}(x, t) d x=M_{0}, I_{1}(t)=I_{2}(t)$ for every $t$. Nevertheless we shall show that if $b_{2}>b_{1}, I_{2}$ is asymptotically larger than $I_{1}$.

We begin by defining in $\left\{(x, t): t>T^{*}, \zeta_{1}(t) \leqslant x \leqslant \zeta_{2}(t)\right\}$ the function

$$
f(x, t)=\nu_{x}(x, t)+\left(x-x^{*}(t)\right) /(m+1) t
$$

(cf. §1.4). We have

$$
\partial f(x, t) / \partial x=\nu_{x x}(x, t)+((m+1) t)^{-1} \geqslant 0
$$

so that $f$ is nondecreasing in $x$. Also

$$
f\left(\zeta_{1}(t), t\right)=-\zeta_{1}^{\prime}(t)-\frac{l(t)}{2(m+1) t}=\frac{d(t)-2 \varepsilon(t)}{2(m+1) t} .
$$

Since $0 \leqslant d(t) \leqslant l_{0}$ we conclude that $f\left(\zeta_{1}(t), t\right)=O(1 / t)$ as $t \rightarrow \infty$. Similarly $f\left(\zeta_{2}(t), t\right)=O(1 / t)$. This and (3.17) give

$$
f(x, t)=O(1 / t) \text { as } t \rightarrow \infty \text { uniformly in } x \in \Omega(t) .
$$

Next we estimate $\nu(x, t)-\tilde{\nu}(x, t)$ for large $t$ : for every $t \geqslant T^{*}$ and $x:\left|x-x^{*}(t)\right|$ $\leqslant r(t)$ we have $\partial(\nu-\tilde{\nu}) / \partial x=f$ so that

$$
\begin{aligned}
|\nu(x, t)-\tilde{\nu}(x, t)| & \leqslant\left|\nu\left(x^{*}(t)-r(t), t\right)\right|+\int_{x^{*}-r}^{x}|f(x, t)| d x \\
& \leqslant \sup \left|\nu_{x}\right| \cdot d(t) / 2+O(1 / t) \cdot r(t)=O(r(t) / t)
\end{aligned}
$$

and the same estimate clearly holds for $\zeta_{1}(t) \leqslant x \leqslant \tilde{\zeta}_{1}(t)$ and $\tilde{\zeta}_{2}(t) \leqslant x \leqslant \zeta_{2}(t)$. (The estimate for $\left|\nu_{x}\right|$ comes from (3.16).) Hence we conclude that

$$
|\nu(x, t)-\tilde{\nu}(x, t)|=O\left(t^{-m /(m+1)}\right) \quad \text { as } t \rightarrow \infty \text { uniformly in } x \in \mathbf{R} .
$$

From this it follows that uniformly in $x$ such that $\left|x-x^{*}(t)\right| \leqslant \alpha r(t), 0<\alpha<1$, if $m>2$ or in $x \in \mathbf{R}$ if $1<m \leqslant 2$ :

$$
|u(x, t)-\tilde{u}(x, t)|=O\left(t^{-2 /(m+1)}\right)
$$

(a simple application of the Mean Value Theorem since $\left.u=((m-1) \nu / m)^{1 /(m-1)}\right)$. 
We are now in a position to estimate $I_{1}$ and $I_{2}$ under the hypothesis that $b_{2}>b_{1}$ : since $\int u(x, t) d x=\int \tilde{u}(x, t) d x$ and $l(t)-\tilde{l}(t)=2 d(t)>0$, the set $G(t)=\{x \in$ $\mathbf{R}: \nu(x, t)<\tilde{\nu}(x, t)\}$ is nonvoid. Let $x_{1}(t)=\inf G(t), x_{2}(t)=\sup G(t)$. We have $\tilde{\zeta}_{1}(t) \leqslant x_{1}(t) \leqslant x_{2}(t) \leqslant \tilde{\zeta}_{1}(t)$. Since

$$
\tilde{\nu}(x, t)=\left(r(t)^{2}-\left|x-x^{*}(t)\right|^{2}\right) / 2(m+1) t
$$

for every $x:\left|x-x^{*}(t)\right| \leqslant r(t)$, recalling (3.14) we obtain, if $\lim d(t)=d>0$, the estimate

$$
x^{*}(t)-x_{1}(t)=o(r(t)),
$$

and the same applies to $x_{2}(t)$. Thus as $t \rightarrow \infty$,

$$
\begin{aligned}
I_{1}(t) & =\int_{[u<\tilde{u}]}|\tilde{u}-u| d x \\
& \leqslant\left|x_{2}(t)-x_{1}(t)\right| \sup _{x_{1}(t) \leqslant x \leqslant x_{2}(t)}|u(x, t)-\tilde{u}(x, t)| \\
& \leqslant o(r(t)) \cdot O\left(r(t)^{-2}\right)=o\left(t^{-1 /(m+1)}\right) .
\end{aligned}
$$

In the following $C$ will stand for any positive constant depending only on $m$. For large $t$ it follows from (3.14), (3.22) that for $r(t) / 3<\left|x-x^{*}(t)\right|<r(t) / 2$,

$$
\nu(x, t)-\tilde{\nu}(x, t) \geqslant C t^{-m /(m+1)},
$$

so that we estimate $I_{+}$from below:

$$
I_{+}(t) \geqslant 2 \cdot(r(t) / 6) \cdot C t^{-2 /(m+1)} \geqslant C t^{-1 /(m+1)} .
$$

(3.24), (3.25) contradict the fact that $I_{+}(t)=I_{-}(t)$ for every $t$. Hence the assumption $b_{2}>b_{1}$ was false. \#

Proof of Theorem A. Part (i) was proved in Corollaries 2.4 and 2.5. To prove part (ii) we repeat the calculations of the preceding lemma, taking into account the fact that $d(t) \downarrow 0$. Thus $x^{*}(t)=b+o(1)$ and (3.18), (3.20), (3.21) give, respectively:

$$
\begin{gathered}
\nu_{x}(x, t)+(x-b) /(m+1) t=o(1 / t) \quad \text { uniformly in } x \in \Omega(t), \\
\nu(x, t)-\bar{\nu}\left(x, t ; M_{0}, b\right)=o\left(t^{-m /(m+1)}\right) \quad \text { uniformly in } x \in \mathbf{R}, \\
u(x, t)-\bar{u}\left(x, t ; M_{0}, b\right)=o\left(t^{-2 /(m+1)}\right),
\end{gathered}
$$

uniformly in $x:|x-b|<\alpha r(t), 0<\alpha<1$, if $m>2$ or in $x \in \mathbf{R}$ if $1<m \leqslant 2$.

It only remains to prove that $b=x_{0}$ :

If $1<m \leqslant 2$ the proof is immediate from (3.28) and the invariance of the center of mass:

$$
\begin{aligned}
M_{0}\left|x_{0}-b\right| & =\left|\int x\left(u(x, t)-\bar{u}\left(x, t ; M_{0}, b\right)\right) d x\right| \\
& =O\left(r(t)^{2}\right) \cdot o\left(t^{-2 /(m+1)}\right)=o(1) .
\end{aligned}
$$

Now let $t \rightarrow \infty$ to obtain $x_{0}=b$. 
Case $m>2$. To simplify the calculation we may assume that $b=0$ (if $b \neq 0$ shift the $x$-axis). Then

$$
\begin{aligned}
M_{0}\left|x_{0}\right|= & \left|\int x(u-\bar{u}) d x\right| \\
\leqslant & \int_{\zeta_{1}(t)}^{-r(t)}|x u(x, t)| d t+\int_{r(t)}^{\zeta_{2}(t)}|x u(x, t)| d t \\
& +\int_{-r(t)}^{r(t)}|x||u(x, t)-\bar{u}(x, t)| d x \\
= & I_{1}+I_{2}+I_{3} .
\end{aligned}
$$

Using (3.27) we estimate $u$ in the region $[|x| \geqslant r(t)]$ as $o\left(t^{-m /\left(m^{2}-1\right)}\right)$ so that

$$
I_{1}+I_{2}=o\left(t^{-m /\left(m^{2}-1\right)}\right) \cdot r(t) \cdot d(t)=o\left(t^{-1 /(m+1)}\right) \rightarrow 0 .
$$

Next we estimate $I_{3}$. By (3.27) there is a function $\varepsilon(t) \geqslant 0, \varepsilon(t) \rightarrow 0$ such that for $|x| \leqslant r(t)$

$$
\bar{\nu}(x, t)-\varepsilon(t) t^{-m /(m+1)} \leqslant \nu \leqslant \bar{\nu}+\varepsilon(t) t^{-m /(m+1)} .
$$

Let $F(t)=\{|x| \leqslant r(t): u(x, t) \geqslant \bar{u}(x, t)\}$. We have $(C$ represents any constant $>$ $0)$ :

$$
\begin{aligned}
& \left|\int_{F(t)} x(u-\bar{u}) d x\right| \leqslant 2 \int_{0}^{r(t)} x C|\nu-\bar{\nu}|^{1 /(m-1)} d x \\
& \quad \leqslant C \int_{0}^{r(t)} x d x\left\{\left|\frac{r(t)^{2}-x^{2}+C \varepsilon(t) r(t)}{2(m+1) t}\right|^{1 /(m-1)}-\left|\frac{r(t)^{2}-x^{2}}{2(m+1) t}\right|^{1 /(m-1)}\right\} d x \\
& \quad \leqslant C r(t)\left\{-C \varepsilon(t) r(t)^{-m /(m-1)}+(1+C \varepsilon(t) / r(t))^{m /(m-1)}-\mathbf{b}\right\} \\
& \quad=o\left(r(t)^{-1 /(m-1)}\right)+o(1) \rightarrow 0 .
\end{aligned}
$$

A similar computation applies on the set $G(t)=\{|x| \leqslant r(t): u(x, t) \leqslant \bar{u}(x, t)\}$ (by replacing $\varepsilon(t)$ by $-\varepsilon(t), \ldots$ ) so that finally $I_{3}(t) \rightarrow 0$ and $x_{0}=0$ (i.e. $x_{0}=b$ ) follows from (3.30) letting $t \rightarrow \infty$. \#

4. Symmetric solutions. In this section we assume that $u_{0}(x)$ is symmetric, i.e.

$$
u_{0}(x)=u_{0}(-x)
$$

Since $(0.1)$ is symmetry-invariant the corresponding solution $u(x, t)$ satisfies

$$
u(x, t)=u(-x, t)
$$

for every $x, t>0$. We set $\zeta(t)=\zeta_{2}(t)=-\zeta_{1}(t)$.

We introduce a comparison principle based on the estimate of the concentration of mass around the origin by means of which we prove optimal rates of convergence 
in the results of Theorem A:

THEOREM B. Let $u(x, t)$ be a solution of $(0.1),(0.2)$ with $u$ satisfying $(0.3),(0.4)$, (4.1). Then there exist $\tau>0$ and $t_{1} \geqslant 0$ such that

$$
\begin{aligned}
& (4.3)=\left(0.10^{\prime}\right) \quad 0 \leqslant(\zeta(t)-r(t)) t^{m /(m+1)} \leqslant k_{1} \cdot M_{0}^{(m-1) /(m+1)} \tau \quad \text { if } t \geqslant t_{1} \text {, } \\
& (4.4)=\left(0.11^{\prime}\right) \quad 0 \geqslant\left(\zeta^{\prime}(t)-r^{\prime}(t)\right) t^{(2 m+1) /(m+1)} \geqslant k_{2} \cdot M_{0}^{(m-1) /(m+1)} \tau \quad \text { if } t \geqslant t_{1} \text {, } \\
& (4.5)=\left(0.12^{\prime}\right) \quad\left|V(x, t)-\frac{x}{(m+1) t}\right| t^{(2 m+1) /(m+1)} \leqslant k_{2} M_{0}^{(m-1) /(m+1)} \tau \\
& \text { if }|x| \leqslant \zeta(t), t \geqslant t_{1}, \\
& (4.6)=\left(0.13^{\prime}\right) \quad \nu(x, t)-\frac{\left[r(t)^{2}-x^{2}\right]}{2(m+1) t}+\mid t^{2 m /(m+1)} \leqslant k_{3} \cdot M_{0}^{2(m-1) /(m+1)} \tau \\
& \text { if } x \in \mathbf{R}, t \geqslant t_{1},
\end{aligned}
$$

for some positive constants $k_{1}=c_{m} /(m+1), \quad k_{2} \leqslant k_{1}((m+1) / m)^{m}, \quad k_{3} \leqslant$ $c_{m}\left(k_{1} /(m+1)+k_{2}\right)$. Moreover there exists $\tau_{1}=\tau_{1}(m)>0$ such that $\tau \leqslant$ $\tau_{1} M_{0}^{1-m} l_{0}^{m+1}$. \#

REMARKS. (1) (4.6) and $u=((m-1) \nu / m)^{1 /(m-1)}$ give the estimate for $u$,

$(4.7)=\left(0.16^{\prime}\right) \quad u(x, t)=\bar{u}\left(x, t: M_{0}\right)+O\left(t^{-(m+2) /(m+1)}\right)$,

if $t \geqslant t_{1}$ uniformly in $x:|x| \leqslant \alpha r(t), 0<\alpha<1$ (if $1<m \leqslant 2$ uniformly in $x \in \mathbf{R}$ ).

(2) The exponents in $\left(0.10^{\prime}\right)-\left(0.14^{\prime}\right)$ are best possible. To see this apply the theorem to the explicit solution $u(x, t)=\bar{u}\left(x, t+\tau ; M_{0}\right)$ for some $\tau>0$, that serves as a model.

(3) We do not treat the problem of determining the best $\tau$ in Theorem B and its consequences. In this respect see the indications in [21].

(4) The proof of Theorem B can be adapted to treat radially-symmetric solutions in spatial dimension $n \geqslant 1$. \#

The proof of Theorem B proceeds by comparing $u$ with the self-similar solutions $\bar{u}\left(x, t ; M_{0}\right)$ and $\bar{u}\left(x, t+\tau ; M_{0}\right)$ for some suitable $\tau>0$. As in $\S 2$ we first prove an elliptic version of the comparison principle.

Lemma 4.1 (Concentration-Comparison Principle. Elliptic Version). Let $\beta$ be a continuous nondecreasing function such that $O=\beta(0) \subset \operatorname{Int} \beta(\mathbf{R})$, let $f_{i}, i=1,2$, be symmetric and integrable functions in $\mathbf{R}$ and define for $r \geqslant 0$,

$$
F_{i}(r)=\int_{|x| \leqslant r} f_{i}(x) d x .
$$

Let $u_{i}, i=1,2$, be the (symmetric) solutions of $-u_{i}^{\prime \prime}+\beta\left(u_{i}\right)=f_{i}[9]$, set $w_{i}=\beta\left(u_{i}\right)$ and for $r>0$,

$$
W_{i}(r)=\int_{|x| \leqslant r} w_{i}(x) d x .
$$

Then if $F_{1}(r) \leqslant F_{2}(r)$ for every $r \geqslant 0$, then $W_{1}(r) \leqslant W_{2}(r)$ for every $r \geqslant 0$. \# 
REMARK. We say that $f_{2}$ is more concentrated than $f_{1}: f_{2} \succ f_{1}$. The lemma implies then that $w_{2}>w_{1}$.

Proof. As in Lemma 2.1 let $G=\left\{r \geqslant 0: W_{1}(r)>W_{2}(r)\right\}$. If $G$ is nonvoid, let $I=(a, b)$ be a maximal interval in $G, 0 \leqslant a<b \leqslant \infty$. As in Lemma $2.1 u_{1}-u_{2}$ is strictly increasing on $I$. Next if $b<\infty$ we have $W_{1}(b)=W_{2}(b), u_{i}(b) \leqslant u_{2}(b)$ arguing as there and we conclude that $a=0$ and $W_{1}(0)>W_{2}(0)$, impossible. The case $b=\infty$ is also similar. \#

Via Semigroup Theory we pass to

Lemma 4.2 (Concentration-Comparison Principle. Parabolic Version). Let $u^{1}(x, t), u^{2}(x, t)$ be solutions of $(0.1),(0.2)$ with symmetric initial data $u_{0}^{1}(x), u_{0}^{2}(x) \in$ $L^{1}(\mathbf{R})$. If for every $r>0$,

$$
\int_{|x| \leqslant r} u_{0}^{1}(x) d x \leqslant \int_{|x| \leqslant r} u_{0}^{2}(x) d x
$$

then for every $t, r>0$ we have

$$
\int_{|x| \leqslant r} u^{1}(x, t) d x \leqslant \int_{|x| \leqslant r} u^{2}(x, t) d x . \quad \#
$$

REMARK. Both lemmas admit obvious $n$-dimensional counterparts valid for radially-symmetric solutions. Hence Theorem $\mathrm{B}$ admits an $n$-dimensional version. \#

COROllaRy 4.3. Let $u(x, t)$ be a solution of $(0.1)-(0.4)$, symmetric with respect to $x$ and assume that there exist $\tau>0, t_{1} \geqslant 0$ such that $u\left(x, t_{1}\right) \succ \bar{u}\left(x, t_{1}+\tau ; M_{0}\right)$. Then for every $t \geqslant t_{1}, u(x, t)>\bar{u}\left(x, t+\tau ; M_{0}\right)$ so that

$$
r(t) \leqslant \zeta(t) \leqslant r(t+\tau) \leqslant r(t)+r(t) \tau /((m+1) t) .
$$

Proof. The inequality $r(t) \leqslant \zeta(t)$ comes from Theorem A. To obtain the inequality $\zeta(t) \leqslant r(t+\tau)$ we use Lemma 4.2 and the fact that $\zeta(t)=$ $\sup \left\{r>0: \int_{|x| \leqslant r} u(x, t) d x<M_{0}\right\}$. \#

We now show that for every $u_{0} \in L^{1}(\mathbf{R})$ satisfying $(0.3),(0.4),(4.2)$ there exist $\tau>0$ and $t_{1} \geqslant 0$ such that (4.12) holds. Moreover we bound above $\tau$ in terms of $M_{0}$ and $l_{0}$ :

LEMma 4.4. There exists $\tau_{1}>0$ such that (4.12) holds with $\tau=\tau_{1} \cdot M_{0}^{1-m} l_{0}^{m+1}$ for all large $t$, and $\tau_{1} \geqslant\left(2 c_{m}\right)^{-(m+1)}$.

Proof. By means of the group of transformations (1.9) we can reduce the proof to the case $M_{0}=l_{0}=1$ : if the lemma is true in this case and $u(x, t)$ is a general solution of $(0.1)-(0.4),(4.2)$, we define $\hat{u}$ by

$$
u(x, t)=\frac{M_{0}}{l_{0}} \hat{u}\left(\frac{x}{l_{0}}, \frac{M_{0}^{m-1}}{l_{0}^{m+1}} t\right) .
$$

We have $\hat{M}_{0}=\hat{l}_{0}=1$ so that $\hat{u}(\cdot, t) \succ \bar{u}\left(\cdot, t+\tau_{1} ; 1\right)$ for all $t \geqslant \hat{t}_{0} \geqslant 0$. Then

$$
u(x, t) \succ \frac{M_{0}}{l_{0}} \bar{u}\left(\frac{x}{l_{0}}, \frac{M_{0}^{m-1}}{l_{0}^{m+1}}+\tau_{1} ; 1\right)=\bar{u}\left(x, t+\frac{\tau_{1} l_{0}^{m+1}}{M_{0}^{m-1}} ; M_{0}\right) .
$$


Therefore we assume that $M_{0}=l_{0}=1$. Now note that there is a worst situation with respect to the relation " $>$ ", namely the one with initial condition

$$
u_{0}(x)=\frac{1}{2} \delta\left(x-\frac{1}{2}\right)+\frac{1}{2} \delta\left(x+\frac{1}{2}\right) .
$$

We only have to prove that there exists a $\tau_{1}$ for this particular $u_{0}$ (the fact that $u_{0}$ is a measure causes no inconvenience, see remark in $\S 1.1)$. Since $u_{0}(x) \geqslant \frac{1}{2} \delta\left(x-\frac{1}{2}\right)$, $u(x, t) \geqslant \bar{u}\left(x-\frac{1}{2}, t ; \frac{1}{2}\right)$ so that for every $t>t_{0}=\left(4 c_{m}^{m+1}\right)^{-1}$ we have $u(x, t) \geqslant$ $\bar{u}\left(\frac{1}{2}, t ; \frac{1}{2}\right)>0$ for $0 \leqslant x \leqslant 1$. Also the free-boundary $\zeta_{2}$ of $\bar{u}$ passes through $\left(1, t_{0}\right)$. By the Sh.C.P. we derive the estimate $1<\zeta(t) \leqslant 1+c_{m}\left(t-t_{0}\right)^{1 /(m+1)}$. To obtain $u(x, t) \succ \bar{u}(x, t+\tau ; 1)$ at a time $t>t_{0}$ we only have to take $\tau$ large enough, for instance such that

$$
\bar{u}(0, t+\tau ; 1) \cdot \zeta(t) \leqslant \bar{u}\left(\frac{1}{2}, t ; \frac{1}{2}\right) .
$$

This $\tau=\tau(t, m)$ is to be minimized in $t>t_{0}$ to obtain $\tau_{1}$.

Consider now the explicit solution $u(x, t)=\bar{u}(x, t+\tau ; 1)$ such that $l_{0}=1$; then $\tau=\left(2 c_{m}\right)^{-(m+1)}$, so that $\tau_{1}>\left(2 c_{m}\right)^{-(m+1)}$. \#

Proof of Theorem B. Corollary 4.3 and Lemma 4.4 imply (4.3).

To prove (4.4) we restrict ourselves as above to the case $M_{0}=l_{0}=1$. Let us estimate the derivative of $\eta(t)=\zeta(t)-r(t)$ using the fact that $t^{m /(m+1)} \eta^{\prime}(t) \uparrow 0$ (Lemma 3.2). For $t>0, \lambda>1$ we have

$$
\begin{aligned}
\eta(t) & =\int_{t}^{\infty}-\eta^{\prime}(t) d t \geqslant \int_{t}^{\lambda t}-\eta^{\prime}(s) d x \geqslant-\eta^{\prime}(\lambda t)(\lambda t)^{m(m+1)} \int_{t}^{\lambda t} s^{-m /(m+1)} d s \\
& =-\eta^{\prime}(\lambda t)(m+1) \lambda^{m /(m+1)} t\left(\lambda^{1 /(m+1)}-1\right) .
\end{aligned}
$$

Using (4.3) we obtain

$$
-\eta^{\prime}(t) \leqslant k_{1} \tau \lambda\left\{(m+1) t^{2(m+1) /(m+1)}\left(\lambda^{1 /(m+1)}-1\right)\right\}^{-1} .
$$

The right-hand expression is minimized setting $\lambda=((m+1) / m)^{m+1}$; then (4.18) gives (4.4) with $k_{1} \leqslant k_{2}((m+1) / m)^{m}$.

Estimates (4.5), (4.6) are obtained by inserting the information (4.3), (4.4) into formulas (3.16)-(3.18) in Lemma 3.3. \#

5. Other results on the growth of the interfaces. In this section we consider solutions $u(x, t)$ of the Cauchy problem $(0.1),(0.2)$, where $u_{0}$ satisfies $(0.3)$, and instead of $(0.4)$ the half-condition

$$
\text { ess } \sup \left(\operatorname{support}\left(u_{0}\right)\right)=0 \text {. }
$$

Then a right free-boundary $x=\zeta(t)$, appears where $\zeta(t)=\{\sup x: u(x, t)>0\}$ for $t>0, \zeta(0)=0$. By the Sh.C.P. $\zeta(t)$ is finite and in fact

$$
0 \leqslant \zeta(t) \leqslant c_{m}\left\|u_{0}\right\|_{1}^{(m-1) /(m+1)} t^{1 /(m+1)} \text {. }
$$

What was said in the Introduction applies and thus there exists a time $t^{*} \geqslant 0$ such that $\zeta(t)=0$ for $0 \leqslant t \leqslant t^{*}$ and $\zeta \in C^{1}\left(\left(t^{*}, \infty\right)\right)$ and $\zeta^{\prime}(t)>0$ if $t>t^{*}$. Also $\zeta(t) t^{-1 /(m+1)}$ and $\zeta^{\prime}(t) t^{m /(m+1)}$ are nondecreasing in $(0, \infty)$. Furthermore we prove

TheOREM C. As $t \rightarrow \infty$ we have (with $\left.r(t)=c_{m}\left(M_{0}^{m-1} t\right)^{1 /(m+1)}\right)$

$$
\zeta(t) / r(t) \uparrow 1, \quad \zeta^{\prime}(t) / r^{\prime}(t) \uparrow 1 .
$$


As $t \rightarrow 0$ we have

$$
\zeta(t) / r(t) \downarrow 0, \quad \zeta^{\prime}(t) / r^{\prime}(t) \downarrow 0 .
$$

Moreover if $x_{0}=M_{0}^{-1} \cdot \int u_{0}(x) x d x,-\infty \leqslant x_{0}<0$,

$$
\zeta(t)-r(t) \downarrow x_{0}
$$

And if $x_{0}$ is finite, then

$$
t\left(\zeta^{\prime}(t)-r^{\prime}(t)\right) \uparrow 0 .
$$

Proof. Take the sequence of approximations to $u(x, t),\left\{u^{n}(x, t)\right\}$ such that $u^{n}(x, t)$ is the solution of $(0.1)$ with initial condition $u_{0}^{n}=u_{0} \cdot \chi_{[-n, 0]}$. If $\zeta^{n}(t)$ is the corresponding right-interface it follows from $u(x, t) \geqslant u^{n}(x, t)$ that $\zeta(t) \geqslant \zeta^{n}(t)$. But by $(2.9) \zeta^{n}(t) t^{-1 /(m+1)} \rightarrow c_{m}\left(M_{n}\right)^{(m-1) /(m+1)}$, where $M_{n}=\int_{-n}^{0} u_{0}(x) d x$. Since $M_{n} \rightarrow M_{0}$ as $n \rightarrow \infty$, this and (5.1) give (5.2). To obtain $\zeta^{\prime}(t) / r^{\prime}(t) \rightarrow 1$ argue as in Lemma 3.2.

To prove (5.3) compare $u$ with the solution $u_{\varepsilon}$ resulting from shifting the initial mass lying in $[-\varepsilon, 0]$, for small $\varepsilon>0$, to 0 as a point mass $M_{\varepsilon} \delta(x)$. In some time interval $\left[0, t_{\varepsilon}\right], t_{\varepsilon}>0$, the right-interface for $u_{\varepsilon}$ coincides with the one for this point mass and the Sh.C.P. gives us

$$
\zeta(t) \leqslant c_{m}\left(M_{\varepsilon}^{m-1} t\right)^{1 /(m+1)} \text { for } 0 \leqslant t<t_{\varepsilon}
$$

Now let $\varepsilon \rightarrow 0$ : then $M_{\varepsilon} \rightarrow 0$ and (5.4) implies that $\zeta(t) / r(t) \rightarrow 0$. Since $\zeta^{\prime}(t) t^{m /(m+1)}$ is monotone the limit $\zeta^{\prime}(t) / r^{\prime}(t)$ exists and is zero.

To prove (5.4) notice that there exists $b,-\infty \leqslant b \leqslant 0$, such that $\zeta(t)-r(t) \downarrow b$ since $\zeta^{\prime}(t) \leqslant r^{\prime}(t)$ for every $t>0$. We shall prove that $b=x_{0}$. For that we call $\hat{u}^{n}(x, t)$ the solution resulting from shifting the mass of $u_{0}$ in $(-\infty, n]$ as a point mass to $x=-n$, keeping $\hat{u}_{0}^{n}(x)=u_{0}(x)$ for $x>-n$. Let $\hat{\zeta}^{n}(t)$ and $\hat{x}_{0}^{n}$ be the corresponding right-interface and center of mass. The sequence $\left\{\hat{\zeta}^{n}(t)\right\}_{n \in N}$ is nonincreasing in $n$ by the Sh.C.P. and Theorem A says that $\hat{\zeta}^{n}(t)=\hat{x}_{0}^{n}+r(t)+o(1)$. Since $\hat{x}_{0}^{n} \downarrow x_{0}$ as $n \rightarrow \infty, b=\lim _{t \rightarrow \infty}(\zeta(t)-r(t)) \leqslant x_{0}$, so that in case $x_{0}=-\infty$ we are done.

It remains to prove that $b \geqslant x_{0}$ in case $x_{0}>-\infty$. Take an $\varepsilon>0$. It is clear that there exist $n_{\varepsilon}$ and $t_{\varepsilon}$ such that for $n \geqslant n_{\varepsilon}$ and $t \geqslant t_{\varepsilon}, \hat{\zeta}^{n}(t)<x_{0}+r(t)+\varepsilon$. Since $\hat{\zeta}^{\prime}(t) / r^{\prime}(t)$ is nondecreasing (we drop the index $n$ in this calculation),

$$
\begin{gathered}
\varepsilon>\hat{\zeta}(t)-r(t)-\hat{x}_{0}=\int_{t}^{\infty}\left(r^{\prime}(s)-\hat{\zeta}^{\prime}(s)\right) d s=\int_{t}^{2 t} r^{\prime}(s)\left[1-\frac{\hat{\zeta}^{\prime}(s)}{r^{\prime}(s)}\right] d s \\
\geqslant(r(2 t)-r(t)) \cdot\left(1-\frac{\hat{\zeta}^{\prime}(2 t)}{r^{\prime}(2 t)}\right)=r(2 t)\left(1-2^{-1 /(m+1)}\right)\left(1-\frac{\hat{\zeta}^{\prime}(2 t)}{r^{\prime}(2 t)}\right) .
\end{gathered}
$$


So that for $t \geqslant 2 t_{\varepsilon}, 1-\hat{\zeta}^{\prime}(t) / r^{\prime}(t) \leqslant k_{m} \varepsilon / r(t)$ with $k_{m}>0$ depending only on $m$. Since $-\hat{\nu}_{x}(\hat{\zeta}(t), t)=\hat{\zeta}^{\prime}(t)[16]$, and $\hat{\nu}_{x x} \geqslant-((m+1) t)^{-1}$, we have at $\bar{x}(t)=x_{0}+r(t)$ $-\varepsilon$, for $t$ large enough $\left(t \geqslant 2 t_{\varepsilon}, 2 k_{m} \varepsilon \leqslant r(t)\right)$ :

$$
\begin{aligned}
\hat{\boldsymbol{v}}(\bar{x}(t), t) & \geqslant\left(r^{\prime}(t)-\frac{k_{m} \varepsilon r^{\prime}(t)}{r(t)}\right)(\hat{\zeta}(t)-\bar{x}(t))-\frac{(\hat{\zeta}(t)-\bar{x}(t))^{2}}{2(m+1) t} \\
& \geqslant \frac{1}{2} r^{\prime}(t) \cdot \varepsilon-\frac{4 \varepsilon^{2}}{2(m+1) t}
\end{aligned}
$$

so that $\hat{\nu}^{n}(\bar{x}(t), t) \geqslant \varepsilon r^{\prime}(t) / 2$ for $t$ large enough uniformly in $n$. Thus in the limit $\nu(\bar{x}(t), t)>0$, so that $\zeta(t) \geqslant \bar{x}(t)$ for every large $t, \lim _{t \rightarrow \infty}\left(\zeta(t)-r(t)-x_{0}\right)>-\varepsilon$ and the result follows.

(5.5) follows from (5.4) arguing as in Lemma 3.2. \#

(5.2) makes clear that for an $u_{0}$ satisfying $(0.3),\left(0.4^{\prime}\right), M_{0}$ and $x_{0}$ allow us to describe $\zeta(t)$ as $t \rightarrow \infty$ in the first approximation. As $t \rightarrow 0(5.3)$ shows that this is not the case: The description of $\zeta(t)$ requires further information: thus Knerr [16] proves that if $u_{0} \in L^{\infty}(\mathbf{R})$ and $\left\|\nu_{0}\right\|_{\infty} \leqslant L\left(\nu_{0}=m u_{0}^{m-1} /(m-1)\right), \zeta(t) \leqslant 2(L t)^{1 / 2}$ for every small $t$ and the exponent $\frac{1}{2}$ is sharp for this class of initial data.

We extend the result to cover the dependence of $\zeta(t)$ on the $L^{p}$-norm of $u_{0}$ for every $1 \leqslant p \leqslant \infty$ : we consider the class of solutions

$$
\begin{aligned}
& e_{p, N}=\{u(x, t): u \text { is solution of }(0.1),(0.2),(0.3),(0.18) \\
& \text { with } \left.u_{0} \in L^{p}(\mathbf{R}) \text { and }\left\|u_{0}\right\|_{p} \leqslant N\right\}
\end{aligned}
$$

where $1 \leqslant p \leqslant \infty, N>0$ and $\|\cdot\|_{p}$ denotes the $L^{p}$-norm. If $\zeta_{u}(t)$ is the rightinterface of $u \in \mathcal{C}_{p, N}$, we set for $t \geqslant 0$,

$$
\mathscr{Z}_{p, N}(t)=\sup \left\{\zeta_{u}(t): u \in \bigodot_{p, N}\right\}
$$

We have

THEOREM D. For every $p: 1 \leqslant p \leqslant \infty$ there exists a constant $C_{p, m}>0$ such that

$$
\mathscr{Z}_{p, N}(t)=C_{p, m}\left(N^{m-1} t\right)^{\alpha}
$$

with $\alpha=p /(2 p+m-1)$ if $1 \leqslant p<\infty, \alpha=\frac{1}{2}$ if $p=\infty$. We have $C_{1, m}=c_{m}\left(c_{m}\right.$ defined in (0.9)).

PROof. By means of the group of transformations $T_{k, L}(\S 1.2)$ we can reduce the proof to the case $N=t=1$. In fact let $u$ be a solution with $L^{p}$-norm $N>0$ and fix a certain $\bar{t}>0$. If we define $\hat{u}$ by

$$
\hat{u}(x, t)=\left(T_{K, L} u\right)(x, t)=K u\left(L x, K^{m-1} L^{2} t\right)
$$

with $L=\left(N^{m-1} \bar{t}\right)^{\alpha}$ and $K=\left(N^{-2 p} \bar{t}\right)^{\beta}$ with $\beta=\alpha / p$ if $p<\infty, \beta=0$ if $p=\infty$, then $\hat{u} \in \bigodot_{p, 1}$ and

$$
\zeta(\bar{t})=L \hat{\zeta}\left(\left(k^{m-1} L\right)^{-1} \bar{t}\right)=\left(N^{m-1} \bar{t}\right)^{\alpha} \cdot \hat{\zeta}(1) .
$$

Hence we must prove that $C_{p, m}=\sup \left\{\zeta_{u}(1): u \in \bigodot_{p, 1}\right\}$ is finite for every $m>1$, $1 \leqslant p \leqslant \infty$. 
For $p=1$ it follows from Corollary 2.3 that $C_{1, m}=c_{m}$. On the other hand for $p=\infty[16]$ proves that $C_{\infty, m} \leqslant 2(m /(m-1))^{1 / 2}$. For $1<p<\infty$ we estimate $C_{p, m}$ in terms of $C_{\infty, m}$ by means of the following " $L^{p}-L^{\infty}$ smoothing effect":

LEMma $5.1|\mathbf{2 0}|$. There exists a positive constant $K=K(m, p)$ such that for every solution of (0.1), (0.2) with $u_{0} \in L^{1}(\mathbf{R}) \cap L^{p}(\mathbf{R}), 1 \leqslant p<\infty$,

$$
\|u(t)\|_{\infty} \leqslant K\left\|u_{0}\right\|_{p}^{\sigma} t^{-\delta}
$$

with $\sigma=2 p /(2 p+m-1), \delta=1 /(2 p+m-1) . \quad \#$

Hence for every $t, h \geqslant 0$ and every $u \in \mathcal{e}_{p, 1}$ we have

$$
\zeta(t+h) \leqslant \zeta(t)+C_{\infty, m}\left(\|u(t)\|_{\infty}^{m-1} t\right)^{1 / 2} .
$$

Fix $t>0$ and set $t_{n}=2^{-n} t, n \geqslant 1$. (5.12) and (5.13) give

$$
\zeta\left(t_{n-1}\right) \leqslant \zeta\left(t_{n}\right)+C_{\infty, m} K^{(m-1) / 2}\left(2^{-n} t\right)^{p /(2 p+m-1)},
$$

and since $\lim _{n \rightarrow \infty} \zeta\left(t_{n}\right)=\zeta(0)=0$, we conclude from (5.14) that

$$
\zeta(t) \leqslant C_{\infty, m}\left(K^{(m-1) / 2} /\left(2^{\alpha}-1\right)\right) t^{\alpha}
$$

with $\alpha=p(2 p+m-1)^{-1}$. Therefore

$$
C_{p, m} \leqslant C_{\infty, m} \cdot K^{(m-1) / 2} \cdot\left(2^{\alpha}-1\right)^{-1} . \quad \#
$$

\section{REFERENCES}

1. D. G. Aronson, Regularity properties of flows through porous media, SIAM J. Appl. Math. 17 (1969), 461-467.

2. Regularity properties of flows through porous media: The interface, Arch. Rational Mech. Anal. 37 (1970), 1-10.

3. D. G. Aronson and $\mathrm{Ph}$. Bénilan, Régularité de l'équation des milieux poreux dans $\mathbf{R}^{N}, \mathrm{C}$. R. Acad. Sci. Paris Sér. A-B 288 (1979), 103-105.

4. D. G. Aronson, L. A. Caffarelli and S. Kamin, How an initially stationary interface begins to move in porous medium flow, Univ. Minnesota Math. Report No. 81-113, 1981.

5. D. G. Aronson and L. A. Peletier, Large-time behaviour of solutions of the porous media equation in bounded domains, J. Differential Equations 39 (1981), 378-412.

6. G. I. Barenblatt, On some unsteady motions of a liquid or a gas in a porous medium, Akad. Nauk SSSR Prikl. Mat Meh. 16 (1952), 67-78. (Russian)

7. G. I. Barenblatt and Ya. B. Zeldovich, The asymptotic properties of self-modelling solutions of the nonstationary gas filtration equations, Soviet Physics Dokl. 3 (1958), 44-47.

8. Ph. Bénilan, Equations d'évolution dans un espace de Banach quelconque et applications, Thesis, Univ. Orsay, 1972.

9. Ph. Bénilan, H. Brézis and M. G. Crandall, A semilinear elliptic equation in $L^{1}\left(\mathbf{R}^{N}\right)$, Ann. Scuola Norm. Sup. Pisa 4 (1975), 523-555.

10. Ph. Bénilan and M. G. Crandall, The continuous dependence on $\phi$ of the solutions of $u_{t}-\Delta \phi(u)=0$, Indiana Univ. Math. J. 30 (1981), 161-177.

11. L. A. Caffarelli and A. Friedman, Regularity of the free-boundary for the one-dimensional flow of gas in a porous medium, Amer. J. Math. 101 (1979), 1193-1218.

12. M. G. Crandall, An introduction to evolution governed by accretive operators, Dynamical Systems-An International Symposium (L. Cesari et al., Editors), Academic Press, New York, 1976, pp. 131-165.

13. A. Friedman and S. Kamin, The asymptotic behaviour of a gas in an n-dimensional porous medium, Trans. Amer. Math. Soc. 262 (1980), 551-563.

14. A. S. Kalashnikov, Formation of singularities in solutions of the equation of nonstationary filtration, $\check{Z}$. Vyčisl. Mat. i Mat. Fiz. 7 (1967), 440-444. 
15. Sh. Kamenomostskaya (Kamin), The asymptotic behaviour of the solution of the filtration equation, Israel J. Math. 14 (1973), 76-78.

16. B. F. Knerr, The porous medium equation in one dimension, Trans. Amer. Math. Soc. 234 (1977), $381-415$.

17. A. A. Lacey, J. R. Ockendon and A. B. Tayler, 'Waiting-time' solutions of a nonlinear diffusion equation, Preprint (1981).

18. O. A. Oleinik, A. S. Kalashinikov and Czhou Yui-Lin, The Cauchy problem and boundary problems for equations of the type of nonstationary filtration, Izv. Akad. Nauk SSSR Ser. Mat. 22 (1958), 667-704. (Russian)

19. L. A. Peletier, The porous media equation, Applications of Nonlinear Analysis in the Physical Sciences (H. Amann et al., Editors), Pitman, London, 1981, pp. 229-241.

20. L. Veron, Coercivité et propriétés régularisantes des semi-groupes non linéaires dans les espaces de Banach, Publ. Fac. Sci. Besançon 3 (1977).

21. Ya. B. Zeldovich and Yu. P. Raizer, Physics of shock-waves and high-temperature hydrodynamic phenomena. Vol. II, Academic Press, New York, 1966, p. 681.

Division de Matematicas, Universidad Autonoma de Madrid, Madrid-34, Spain

Current address: School of Mathematics, University of Minnesota, Minneapolis, Minnesota 55455 